\title{
Adenosine Stress Perfusion Cardiac MRI: Improving Image Quality Using a 32-Channel Surface Coil
}

\author{
Thomas R. Burchell, Redha Boubertakh, Saidi Mohiddin, Marc E. Miquel, \\ Mark A. Westwood, Anthony Mathur, L. Ceri Davies \\ The London Chest Hospital, London, UK \\ E-mail: ceri.davies@bartsandthelondon.nhs.uk \\ Received September 14, 2011; revised November 8, 2011; accepted November 18, 2011
}

\begin{abstract}
Purpose: Adenosine stress CMR is commonly used to assess myocardial ischaemia. Obtaining high quality images requires maximising signal to noise ratio (SNR) over a large double-oblique field of view (FOV) whilst minimising artefacts. A 32-channel surface coil may provide a higher SNR over a larger FOV compared to standard coils, possibly leading to improved image quality. Materials and Methods: 50 adenosine perfusion CMR scans were performed on a Philips Achieva CV 1.5T, with either a 5 or 32-channel coil (25 patients each) using standardised acquisition protocols. 3 short axis slices were acquired per cardiac cycle and the resulting cine images were scored by two blinded CMR specialists on a quality scale of 1 to 5 . Phantom studies were performed using similar acquisition parameters and the SNR was calculated and compared across a range of acceleration factors. Results: The mean patient age was $62 \pm 11$ years and 50\% of patients were male. The image quality scores were higher using the 32-channel coil (mean $3.8 \pm 0.7$ vs $3.2 \pm 0.9 \mathrm{p}=$ 0.002). The average phantom SNR was greater for the 32-element coil across the range of acceleration factors measured ( 103 vs $86 \mathrm{p}=<0.001$ ). Conclusions: The 32-channel coil produces significantly higher quality images and a higher SNR than the 5-channel coil in routine perfusion CMR.
\end{abstract}

Keywords: Cardiovascular MRI, Myocardial Perfusion, 32-Channel Coil, Adenosine

\section{Introduction}

\section{Perfusion Imaging}

First pass stress perfusion cardiac magnetic resonance imaging (Stress Perfusion CMR) has been comprehensively shown to be a safe, reliable and reproducible method of identifying areas of inducible myocardial ischaemia without the use of ionising radiation [1-5]. It has also been shown to predict cardiovascular morbidity and mortality in patients with known coronary disease [6].

Acquiring high quality diagnostic images in a routine clinical setting can be technically challenging due to the limitations of the current hardware and acquisition protocols. These limitations can lead to a low in-plane resolution, resulting in dark rim Gibbs artefact [7], non-uniform sensitivity over the FOV, poor SNR and long acquisition times.

Various techniques have been used in an attempt to overcome these limitations:

A combination of highly accelerated parallel imaging and temporal encoding (e.g. $k$ - $t$ SENSE) can be used to increase spatial resolution $[8,9]$ however, using temporal information requires post processing and is prone to movement artefacts, which cannot be identified until after the perfusion scan has been completed. Higher field strengths to increase contrast to noise ratio (CNR) have shown promise using $3 \mathrm{~T}$ but CNR inhomogeneities occur across the left ventricle, particularly in the inferior wall [10]. Also 3T magnets are not yet widely available in the clinical setting.

Increasing the number of surface coils has the potential advantages of increasing the SNR [11-13] and provides a better field of view coverage, allowing higher acceleration factors and hence higher spatial resolution or faster acquisition times [14].

We assessed the difference in visual quality and SNR between dedicated cardiac 32-element and standard 5element surface coils in routine clinical myocardial perfusion imaging using an identical acquisition protocol for both coils. 


\section{Materials and Methods}

All scans were performed using a Philips Achieva CV 1.5T MR scanner providing a gradient strength of 33 $\mathrm{mT} / \mathrm{m}$ and maximum slew rate of $180 \mathrm{mT} / \mathrm{m} / \mathrm{ms}$ (Philips Medical Systems, Best, The Netherlands). The scanner was equipped with 32 independent receiver channels. The scans were performed with either a standard 5-element (Philips) or 32-element phased-array surface coil (In vivo Corporation, Gainesville, FL, USA) (Figure 1).

The 32-element coil consists of two parts, an anterior (flexible) and posterior (fixed), each with 16 elements, which are $9.1 \times 10.6 \mathrm{~cm}$ in size and are overlapped to prevent mutual inductance. Both arrays are arranged in a $4 \times$ 4 honeycomb pattern and measure $30.3 \times 36.4 \mathrm{~cm}$ overall, being widest in the left-right direction. The standard 5-element phased-array coil also consists of 2 parts, with a fixed posterior 3-element and flexible anterior 2element coil. The anterior circular coils are $200 \mathrm{~mm}$ in diameter and overlap, the posterior array is made of three rectangular coils measuring $138 \times 200 \mathrm{~mm}$ (Figure 1).

\subsection{Declaration of Helsinki}

This study was conducted in accordance with the princeples of the "Declaration of Helsinki" (as amended in Tokyo, Venice, Johannesburg, and Edinburgh) and within the local laws and regulations.

\subsection{Patient Studies}

\subsubsection{Patients}

50 consecutive patients ( 25 with each coil) through the routine clinical service underwent perfusion CMR scans. They were instructed to refrain from any caffeine containing products for $>12$ hours prior to the scan. Both the

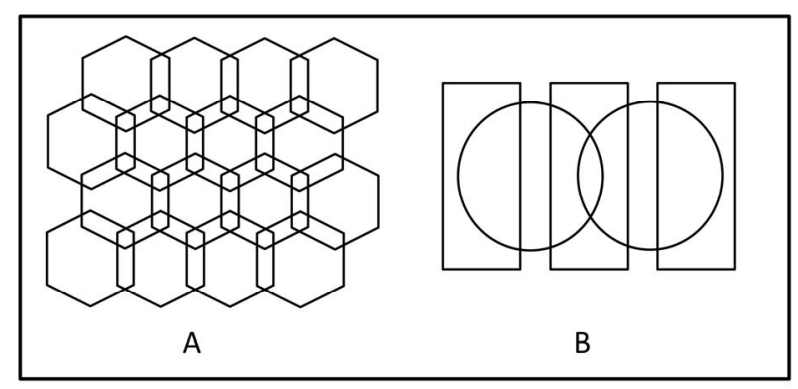

Figure 1. 5 and 32-element receiver coils. A schematic drawing of the receiver coils. Pane A shows the posterior section of the 32-element phased array coil, demonstrating the 16 hexagonal overlapping coil elements. The anterior part uses a further 16 elements in the same configuration (not shown for clarity). Pane B shows the anterior 2 circular elements and the posterior 3 rectangular elements of the 5-element coil. acquisition protocols and the image manipulation/analyses were identical for both surface coils).

\subsubsection{Stress Protocol}

Intravenous adenosine was infused at $140 \mu \mathrm{g} / \mathrm{Kg} / \mathrm{min}$ for 3 minutes with continuous heart rate and blood pressure recording every minute. Following this, an intravenous bolus of $0.05 \mathrm{mmol} / \mathrm{kg}$ of Gadoteric acid (Dotarem, Guerbet, France) was administered via an antecubital fossa vein on the contralateral arm to the adenosine, using a power injector (Spectris Solaris EP, Medrad, Indianola, PA, USA) at a rate of $4 \mathrm{ml} / \mathrm{sec}$ with an immediate $0.9 \%$ saline flush of $25 \mathrm{ml}$ at a rate of $4 \mathrm{ml} / \mathrm{sec}$.

3 short axis slices, each of $10 \mathrm{~mm}$ thickness, were acquired per cardiac cycle, at the basal, mid papillary and apical levels of the left ventricle, with the patient freebreathing throughout the acquisition. We used a single shot prospectively gated, balanced steady state free precession sequence (TR $2.6 \mathrm{~ms}$ TE $1.3 \mathrm{~ms}$, Flip angle 50) and typical voxel size of $2.8 \times 2.9 \times 10 \mathrm{~mm}$ with a matrix size of $111-131 \times 256-320$, with a field of view (FOV) between $343 \mathrm{~mm}$ and $410 \mathrm{~mm}$ to minimise aliasing artefacts. A saturation pre-pulse was applied prior to data acquisition. A 90 degrees preparation pulse was used with a $100 \mathrm{~ms}$ recovery time. This saturation pulse was repeated before the acquisition of every slice within a cardic cycle. Parallel imaging was used with a sensitiveity encoding (SENSE) factor of 2.1 - 2.5.

\subsubsection{Image Manipulation and Analysis}

The 3 stress cine images were saved as lossless avi videos: Codec: Microsoft MPEG-4 V2, Resolution: $1250 \times$ 925, Colour Depth: 16 million, Frame rate: 13fps.

The videos were then reviewed independently, by two experienced cardiac MRI specialists (who were blinded to the choice of coil) in a randomised and anonymised sequence, using Windows Media Player (Microsoft, Seattle, USA). Each video was assessed for image quality, noise and artefact and given an overall visual quality score using a 5 point scale, where 1 = Non Diagnostic; 2 = Poor; 3 = Adequate; 4 = Good; 5 = Excellent.

\subsection{Phantom Study}

Measurements were performed for both coils, using a rectangular phantom filled with a $\mathrm{CuSO}_{4} / \mathrm{NaCl}$ solution (770 mg $\mathrm{CuSO}_{4}+2000 \mathrm{mg} \mathrm{NaCl} / 1000 \mathrm{ml}$ of water) and with dimensions of $260 \mathrm{~mm} \times 240 \mathrm{~mm} \times 370 \mathrm{~mm}(\mathrm{~L} \times$ $\mathrm{W} \times \mathrm{H}$ ), in a sagittal oblique orientation, using a non balanced gradient echo sequence, with the same scan parameters used for human studies with the exception of a saturation pulse. Reconstruction filters were disabled to assess the non-smoothed reconstructed images. A range 
of SENSE factors were acquired: 1.0, 1.5, 2.0, 2.3, 2.5, 2.7, 3.0, 3.5 and 4.0, with the majority between 2 and 3 to mirror clinical practice. Measurements were repeated 100 times for each sequence to allow for a temporal mean to be calculated. The noise standard deviation was assessed on magnitude images using the multiple acquisition method [13]. To compensate for the Rayleigh noise distribution in modulus images, a correction factor of 0.655 was used to correct the underestimated noise standard deviation. The mean signal (MS) and the standard deviation (SD) were measured. From this data, the signal to noise ratio (SNR) was calculated as previously described [15]. A central circular region of interest, measuring $110 \mathrm{~mm}$, covering approximately $20 \%$ of the total phantom area was used for analysis, in a location similar to the heart size and position (see Figure 2).

\subsection{Statistics}

The image quality scores for the 5 and 32-channel coils were compared using the Mann-Whitney test for both observers individually and also with their scores combined. Intra-observer variability was assessed by comparing the different observer scores, using Bland Altman analysis. Signal to noise values were compared using a Student's t-test. All p-values are two tailed. Statistical analysis was performed using SPSS Version 17.0 (SPSS Inc, Chicago, IL, USA).

\section{Results}

\subsection{Patient Studies}

Patient characteristics are listed in Table 1. The mean

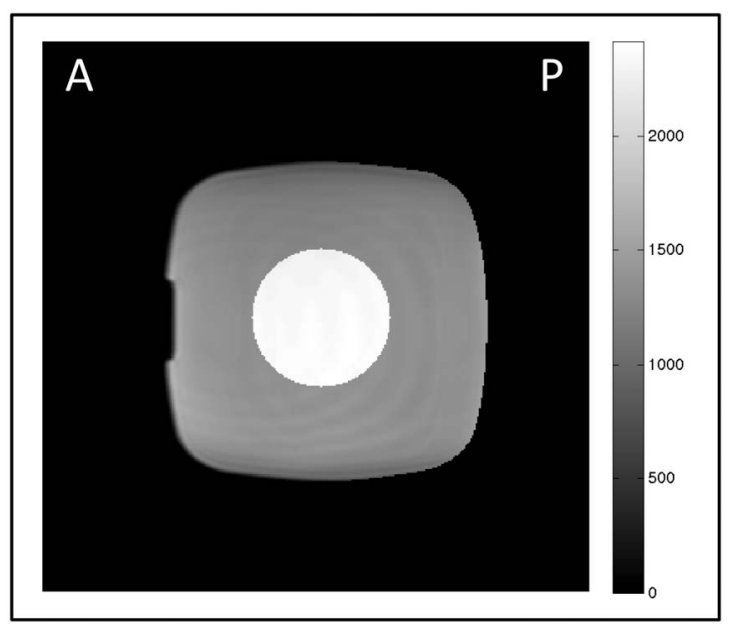

Figure 2. Phantom measurements. Sagittal oblique phantom cross section. The phantom's central area (white circular ROI) is used for signal to noise and standard deviation measurements for both 5 and 32 channel coils. patient age was $62 \pm 11$ years (range 36 to 82 years), and 25 (50\%) were male. There was an improvement in image quality score using the 32-channel coil compared to the 5-channel coil for observer 1 (mean score $4.1 \pm 0.7$ vs. 3.5 $\pm 1 \mathrm{p}=0.04$ ), observer 2 (mean score $3.4 \pm 0.7$ vs. $3.0 \pm$ $0.6 \mathrm{p}=0.02$ ) and with both observers' scores combined (mean score $3.8 \pm 0.7$ vs. $3.2 \pm 0.9 \mathrm{p}=0.002$ ), which was highly statistically significant. The mean difference in scores between observer 1 and 2 was $0.6 \pm 1.7$ (Figure 3 ).

\subsection{Phantom Study}

The minimum, maximum and mean SNR values for both coils are listed in Table 2 . The average SNR was greater for the 32-element coil than the 5-element coil across the entire range of acceleration factors measured from 1 to 4 (103 vs $86 \mathrm{p}=<0.001)$ (Figure 4).

\section{Discussion}

There has been a recent and rapid increase in the demand for myocardial perfusion assessment, especially for planning reperfusion strategies [16,17].

CMR perfusion imaging has been shown to be non-inferior to SPECT in demonstrating myocardial ischaemia [18] and has the added advantages of providing validated information about both left ventricular function and viability as well as being free of ionising radiation.

CMR perfusion, however is still constrained by technical limitations. Each image is acquired over a relatively short period of the cardiac cycle as a single shot, yielding a low SNR, resulting in a relatively low resolution.

Ideally, a high quality perfusion study depends on a number of factors, including; high image resolution, adequate cardiac coverage, a high signal to noise ratio and minimal artefact.

This study has demonstrated that the 32-channel coil provides superior observed image quality for qualitative myocardial perfusion scanning. This is due mainly to increased SNR and the larger FOV coverage afforded by this design of phased array coil.

The SNR was shown (in a phantom) to be significantly higher for the 32-channel coil over a wide range of ac-

Table 1. Baseline characteristics of the study population.

\begin{tabular}{cccc}
\hline Characteristics & 5-channel & 32-channel & p-value \\
\hline Age & $61.5 \pm 10.4$ & $62.3 \pm 11.4$ & 0.80 \\
Gender & 11 male (44\%) & 14 male (56\%) & 0.57 \\
Weight in kg & $79.5 \pm 13.9$ & $72.2 \pm 9.6$ & 0.035 \\
$\begin{array}{c}\text { Peak HR } \\
\text { (during stress) }\end{array}$ & $89.7 \pm 20.9$ & $92.0 \pm 24.3$ & 0.71 \\
\hline
\end{tabular}


Table 2. Signal to noise ratios.

\begin{tabular}{ccccccc}
\hline & \multicolumn{3}{c}{ SNR 5-CH } & \multicolumn{3}{c}{ SNR-32-CH } \\
\cline { 2 - 7 } SENSE & Mean & Max & Min & Mean & Max & Min \\
\hline $\mathbf{1 . 0}$ & 140.4 & 217.0 & 96.0 & 151.8 & 233.7 & 109.6 \\
$\mathbf{1 . 5}$ & 112.4 & 176.7 & 75.7 & 128.2 & 182.8 & 87.8 \\
$\mathbf{2 . 0}$ & 98.6 & 160.4 & 60.8 & 108.1 & 171.1 & 71.7 \\
$\mathbf{2 . 3}$ & 87.1 & 131.1 & 54.9 & 100.5 & 150.5 & 73.8 \\
$\mathbf{2 . 5}$ & 86.5 & 140.9 & 53.3 & 98.3 & 145.9 & 66.7 \\
$\mathbf{2 . 7}$ & 79.0 & 145.7 & 49.2 & 94.7 & 140.3 & 64.8 \\
$\mathbf{3 . 0}$ & 72.4 & 99.9 & 45.7 & 91.7 & 133.4 & 61.0 \\
$\mathbf{3 . 5}$ & 51.9 & 85.4 & 25.3 & 76.8 & 117.2 & 39.6 \\
$\mathbf{4 . 0}$ & 42.5 & 72.3 & 20.7 & 77.7 & 120.5 & 41.4 \\
\hline
\end{tabular}

Signal to noise ratios for each coil over a range of acceleration factors. Results are expressed as mean, maximum and minimum values within the central region of interest for each coil and each acceleration factor. Note: there is a greater number of measurements between acceleration factors 2 to 3 to mirror standard clinical practice.

celeration factors, most importantly in the range of common clinical practice $(\mathrm{R}=2$ - 3).

This increased SNR should allow the use of higher acceleration factors whilst minimising the noise degradation seen with the use of standard coils. Speeding up the acquisition in this way would facilitate either an increase in cardiac coverage (more slices) or a higher image resolution, reducing dark rim artefact [9], which is an area of potential further investigation.

Routine use of the 32-channel coil could provide images that are easier and faster to interpret by the physiccian and may improve workflow through the MRI de-

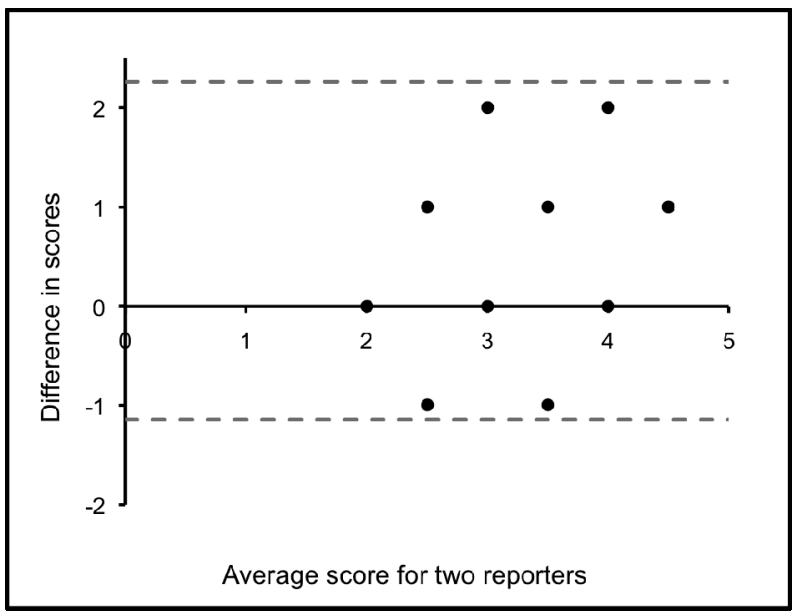

Figure 3. Intra-observer variability. Bland Altman plot showing variance from the mean for the 2 observer's scores. The 0.95 confidence intervals $( \pm 1.96$ standard deviations) are shown as dashed lines.

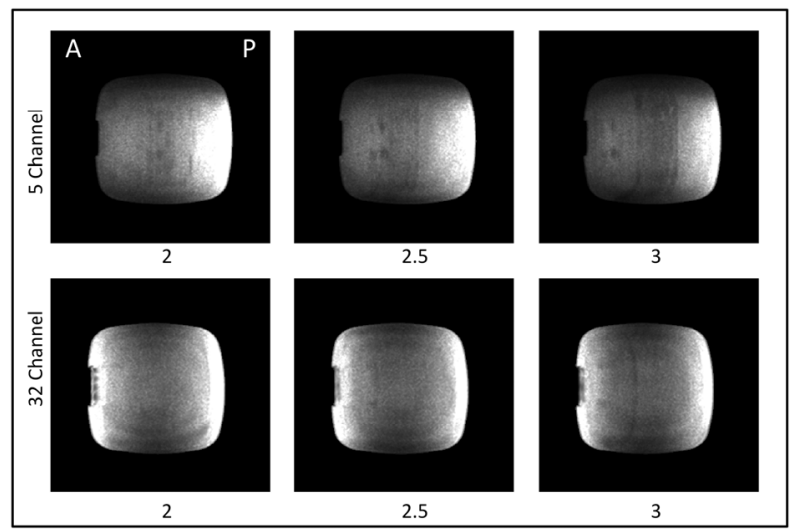

Figure 4. SNR maps. Signal to noise for the 5 channel coil (top row) and 32 channel coil (bottom) for 3 SENSE acceleration factors. The phase encoding direction is AP.

partment. By extension of the increase in SNR and image quality, they may prove to have a higher sensitivity and specificity, however this is beyond the scope of this study.

Hardware limitations:

The 32-channel coil however is not without limitations: As it is still a new technology this coil is not currently in widespread use. It is considerably larger in all dimensions than the standard coil and weighs approximately $1.2 \mathrm{~kg}$ more, leading to an effective reduction in bore size and an increased sensation of confinement. The subsequent increased coverage of the coil may however, reduce the dependence on coil positioning, especially when imaging in the double oblique plane.

Study Limitations:

The study used unpaired patient groups, as it was an observation of a real world clinical service. As a conesquence, there is a small difference in weights between the groups. Although significantly increased body weight may impact on image quality, the difference in groups was less than $10 \%$. The phantom data provides a paired comparison between the 2 coil types. The clinical data was included as an indicator of the clinical utility of increased image quality.

Image quality is a subjective measurement, being an assessment of a combination of noise, artefact, resolution and contrast, although in our observations the variation between observer scores was within the $95 \%$ confidence interval.

We have not indicated whether the improved image quality translates into improved identification of perfusion defects, which will require further investigation and this is clearly a significant weakness. Assessment of test sensitivity and specificity would require an invasive coronary angiogram in all patients, which would not be normally indicated in the presence of a normal functional study. 
In conclusion, the 32-channel phased array coil produces both a higher SNR and significantly higher quality images than the standard 5-channel coil in routine stress CMR despite using near identical acquisition protocols for both coils.

\section{References}

[1] N. Al-Saadi, E. Nagel, M. Gross, et al., "Noninvasive Detection of Myocardial Ischemia from Perfusion Reserve Based on Cardiovascular Magnetic Resonance," Circulation, Vol. 101, No. 12, 2000, pp. 1379-1383.

[2] T. Giang, D. Nanz, R. Coulden, et al., "Detection of Coronary Artery Disease by Magnetic Resonance Myocardial Perfusion Imaging with Various Contrast Medium Doses: First European Multi-Centre Experience,” European Heart Journal, Vol. 25, No. 18, 2004, pp. 16571665. doi:10.1016/j.ehj.2004.06.037

[3] E. Nagel, C. Klein, I. Paetsch, et al., "Magnetic Resonance Perfusion Measurements for the Noninvasive Detection of Coronary Artery Disease," Circulation, Vol. 108, No. 4, 2003, pp. 432-437. doi:10.1161/01.CIR.0000080915.35024.A9

[4] J. Schwitter, D. Nanz, S. Kneifel, et al., “Assessment of Myocardial Perfusion in Coronary Artery Disease by Magnetic Resonance a Comparison with Positron Emission Tomography and Coronary Angiography," Circulation, Vol. 103, No. 18, 2001, pp. 2230-2235.

[5] N. Wilke, M. Jerosch-Herold, A. Zenovich and A. Stillman, "Magnetic Resonance First-Pass Myocardial Perfusion Imaging: Clinical Validation and Future Applications," Journal of Magnetic Resonance Imaging, Vol. 10, No. 5, 1999, pp. 676-685.

doi:10.1002/(SICI)1522-2586(199911)10:5<676::AID-J MRI10>3.0.CO;2-L

[6] C. Jahnke, E. Nagel, R. Gebker, et al., "Prognostic Value of Cardiac Magnetic Resonance Stress Tests: Adenosine Stress Perfusion and Dobutamine Stress Wall Motion Imaging," Circulation, Vol. 115, No. 13, 2007, pp. 17691776. doi:10.1161/CIRCULATIONAHA.106.652016

[7] E. Di Bella, D. Parker and A. Sinusas, "On the Dark Rim Artifact in Dynamic Contrast-Enhanced MRI Myocardial Perfusion Studies," Magnetic Resonance in Medicine, Vol. 54, No. 5, 2005, pp. 1295-1299.

[8] T. Niendorf and D. Sodickson, "Highly Accelerated Cardiovascular MR Imaging Using Many Channel Technology: Concepts and Clinical Applications,” European Radiology, Vol. 18, No. 1, 2008, pp. 87-102. doi:10.1007/s00330-007-0692-0

[9] S. Plein, S. Kozerke, D. Suerder, et al., "High Spatial
Resolution Myocardial Perfusion Cardiac Magnetic Resonance for the Detection of Coronary Artery Disease,” European Heart Journal, Vol. 29, No. 17, 2008, pp. 2148- 2155.

[10] P. Araoz, J. Glockner, K. McGee, et al., “3 Tesla MR Imaging Provides Improved Contrast in First-Pass Myocardial Perfusion Imaging over a Range of Gadolinium Doses," Journal of Cardiovascular Magnetic Resonance, Vol. 7, No. 3, 2005, pp. 559-564. doi:10.1081/JCMR-200060622

[11] C. J. Hardy, H. E. Cline, R. O. Giaquinto, et al., "32Element Receiver-Coil Array for Cardiac Imaging,” Magnetic Resonance in Medicine, Vol. 55, No. 5, 2006, pp. 1142-1149. doi:10.1002/mrm.20870

[12] Y. Zhu, C. J. Hardy, et al., "Highly Parallel Volumetric Imaging with a 32-Element RF Coil Array,” Magnetic Resonance in Medicine, Vol. 52, No. 4, 2004, pp. 869877. doi:10.1002/mrm.20209

[13] S. Reeder, B. Wintersperger, O. Dietrich, et al., "Practical Approaches to the Evaluation of Signal-to-Noise Ratio Performance with Parallel Imaging: Application with Cardiac Imaging and a 32-Channel Cardiac Coil,” Magnetic Resonance in Medicine, Vol. 54, No. 3, 2005, pp. 748-754. doi:10.1002/mrm.20636

[14] M. Ohliger and D. Sodickson, "An Introduction to Coil Array Design for Parallel MRI," NMR in Biomedicine, Vol. 19, No. 3, 2006, pp. 300-315. doi:10.1002/nbm.1046

[15] M. Fenchel, V. Deshpande, K. Nael, et al., "Cardiac Cine Imaging at 3 Tesla: Initial Experience With a 32-Element Body-Array Coil,” Investigative Radiology, Vol. 41, No. 8, 2006, pp. 601-608. doi:10.1097/01.rli.0000223896.70095.49

[16] L. Shaw, D. Berman, D. Maron, et al., “Optimal Medical Therapy with or without Percutaneous Coronary Intervention to Reduce Ischemic Burden: Results from the Clinical Outcomes Utilizing Revascularization and Aggressive Drug Evaluation (COURAGE) Trial Nuclear substudy,” Circulation, Vol. 117, No. 10, 2008, pp. 12831291. doi:10.1161/CIRCULATIONAHA.107.743963

[17] P. Tonino, B. De Bruyne, N. Pijls, et al., "Fractional Flow Reserve versus Angiography for Guiding Percutaneous Coronary Intervention," The New England Journal of Medicine, Vol. 360, No. 3, 2009, pp. 213-224. doi:10.1056/NEJMoa0807611

[18] J Schwitter, C. Wacker, A. van Rossum, et al., "MRIMPACT: Comparison of Perfusion-Cardiac Magnetic Resonance with Single-Photon Emission Computed Tomography for the Detection of Coronary Artery Disease in a Multicentre, Multivendor, Randomized Trial," European Heart Journal, Vol. 29, No. 4, 2008, pp. 480-489. doi:10.1093/eurheartj/ehm617 